\title{
A CONNECTED AND REGULAR POINT SET WHICH HAS NO SUBCONTINUUM*
}

\author{
BY \\ R. L. WILDER
}

It is well known $\dagger$ that every closed, connected and regular (connected im kleinen) $\ddagger$ point set is arc-wise§ connected. That a connected and regular point set which is not closed is not necessarily arc-wise connected, and, indeed, may contain no arc, was shown by R. L. Moore.\| Since an arc is a very special kind of continuum, १ Moore's result suggests the possibility of the existence of connected and regular point sets which not only fail to contain any arc, but do not contain any continuum whatsoever. It is the purpose of this paper to establish the existence of such sets.

I shall make use of an example of a connected set $S$ containing a point $a$ such that $S-a$ is totally disconnected, given by B. Knaster and C. Kuratowski.** For the sake of brevity, I shall assume familiarity with this set as well as with the proof of its connectivity.

\section{I}

Before proceeding to the construction of the point set which I wish to present in this paper, I shall first construct, and establish certain properties

* Presented to the Society, April 2, 1926; received by the editors in May, 1926.

$\dagger$ Cf. R. L. Moore, A theorem concerning continuous curves, Bulletin of the American Mathematical Society, vol. 23 (1917), pp. 233-236; S. Mazurkiewicz, Sur les lignes de Jordan, Fundamenta Mathematicae, vol. 1 (1920), pp. 166-209; H. Tietze, Über stetige Kurven, Jordansche Kurvenbogen und geschlossene Jordansche Kurven, Mathematische Zeitschrift, vol. 5 (1919), pp. 284-291.

$\ddagger$ A point set $M$ is said to be regular or connected im kleinen at a point $P$ if for every circle $K$ with center at $P$ there exists a circle $R$ concentric with $K$ such that if $x$ is a point of $M$ interior to $R$, then $P$ and $x$ both lie in a connected subset of $M$ which lies wholly interior to $K$. The set $M$ is said to be regular or connected im kleinen if it is regular at every one of its points. Cf. H. Hahn, Über die allgemeinste ebene Punktmenge, die stetiges Bild einer Strecke ist, Jahresbericht der Deutschen Mathematiker-Vereinigung, vol. 23 (1924), pp. 318-322. Also S. Mazurkiewicz, loc. cit.

$\S$ A point set $M$ is said to be arc-wise connected if every two of its points are the end points of at least one $\operatorname{arc}$ of $M$.

\| See Bulletin of the American Mathematical Society, vol. 29 (1923), p. 438.

If A continuum is a closed and connected point set which contains more than one point.

** Sur les ensembles connexes, Fundamenta Mathematicae, vol. 2 (1921), pp. 206-255. The set $S$ referred to here is described on pp. 241-244. Hereafter, I shall refer to this paper as S.E.C. 
of, a set of which a certain important subset is in one-to-one continuous correspondence with the set $S-a$.

On the interval $[0,1]$ of the $x$-axis, let $X$ be a non-dense perfect set. On all lines $x=\xi$, where $\xi$ is the abscissa of an end point of an interval complementary to $X$, consider those points whose ordinates are rational numbers $y$ such that $0 \leqq y<1$. Call the set of all such points $P_{1}$. Let $P_{2}$ be the set consisting of all points whose ordinates are irrational numbers between 0 and 1 on lines $x=\xi$, where $\xi$ is the abscissa of a point of $X$ which is not an end point of an interval complementary to $X$.

There exists a one-to-one continuous correspondence between the set $P_{1}+P_{2}$ and the set $S-a$, which may be defined in the following manner: Let the set $X$ described above and the set $C$ as defined in $\$ 5$ of S.E.C. be identical. For each point $P$ of $S-a$ let $x_{P}$ denote the abscissa of the point in which the $x$-axis is intersected by the line from $P$ to $a$ and let $y_{P}$ denote twice the ordinate of $P$. There exists a correspondence between $S-a$ and $P_{1}+P_{2}$ in which to each point $P$ of $S-a$ there corresponds the point of $P_{1}+P_{2}$ whose abscissa and ordinate are $x_{P}$ and $y_{P}$ respectively. This correspondence is continuous and will hereafter be called the correspondence $Z$.

As the segments* complementary to $X$ form a denumerable set, they can be ordered in a sequence $e_{1}, e_{2}, e_{3}, \cdots$. The set of all points which lie above $e_{n}(n=1,2,3, \cdots)$ and below $y=1$ denote by $s_{n}$. Let $Q_{j}, j=2^{n-1}$, $2^{n-1}+1, \cdots, 2^{n}-1$, be $2^{n-1}$ connected sets such that if $L_{i}$ is the line $y=i / 2^{n}$, where $i$ takes on all odd integral values between 0 and $2^{n}$, and $P_{1 \text { in }}$ and $P_{2 \text { in }}$ are the end points of that segment of $L_{i}$ whose projection on the $x$-axis is $e_{n}$, then for $j=2^{n-1}+\frac{1}{2}(i-1)$, (1) $Q_{j}$ contains $P_{1 i n}$ and $P_{2 i n}$, is regular at these points, and, except for these two points, lies wholly in $s_{n}$; (2) all the limit points of $Q_{j}$ except $P_{1 i n}$ and $P_{2 i n}$ lie in $s_{n}$; (3) if $x$ is any point of $Q_{j}$, the distance of $x$ from $L_{i}$ is less than $1 / 2^{n}$.

Let

$$
M=P_{1}+P_{2}+\sum_{i=1}^{\infty} Q_{i}
$$

Definition. If $A$ and $B$ are two distinct points of a point set $N$, then $A$ and $B$ are separated in $N$ in the weak sense provided $N$ contains no connected subset which contains both $A$ and $B$. The points $A$ and $B$ are sepa-

* By segment I mean an interval without its end points.

$\dagger$ This number is purely conventional, since all that is necessary for the proof below is that the diameter of $Q_{j}$ approach zero as $j$ increases. 
rated in $N$ in the strong sense if there exist two mutually separated* sets whose sum is $N$ and which contain $A$ and $B$, respectively.

The following lemma is part of a theorem which $I$ have established in another connection, but which is not yet published:

LEMMA 1. If $N$ is a continuous curve, $\dagger$ and $A$ and $B$ are two distinct points of an open subset $K$ of $N$ which are not separated in $K$ in the strong sense, then $A$ and $B$ are not separated in $K$ in the weak sense.

LEMMA 2. Let $J$ be a plane simple closed curve which is the sum of two arcs $A x B$ and $A y B$ which have only their end points $A$ and $B$ in common, $R$ the bounded domain complementary to $J$, and $C$ a continuum which separates the plane between $A$ and $B . \ddagger$ Then there exists a continuum $C^{\prime}$ which is a subset of $C$ and of $J+R$, and which has at least one point in common with each of the arcs $A x B$ and $A y B$.

Suppose no such continuum as $C^{\prime}$ exists. For every point $P$ of $C$ in $R$ let $M(P)$ denote that maximal connected subset $\S$ of $C \times(J+R)$ determined by $P$. Every set $M(P)$ has at least one point in common with one of the arcs $A x B$ and $A y B \|$ but not with both. Every point $P$ whose corresponding $M(P)$ contains a point of $A x B$ assign to a set $M_{1}$, and every point $P$ whose corresponding $M(P)$ contains a point of $A y B$ assign to a set $M_{2}$. There exists on $A x B$ an arc $A^{\prime} x B^{\prime}$ whtch does not contain $A$ or $B$, but which does contain every point of the set common to $C$ and $A x B$. Similarly there exists on $A y B$ an $\operatorname{arc} A^{\prime \prime} y B^{\prime \prime}$ which does not contain $A$ or $B$, but which contains every point common to $C$ and $A y B$.

Let

$$
\begin{aligned}
A^{\prime} x B^{\prime}+M_{1} & =N_{1}, \\
A^{\prime \prime} y B^{\prime \prime}+M_{2} & =N_{2} .
\end{aligned}
$$

$N_{1}$ and $N_{2}$ are continua neither of which separates $A$ from $B$ in $J+R$.

\footnotetext{
* Two point sets are said to be mutually separated if they are mutually exclusive and neither contains a limit point of the other.

† A continuous curve is a bounded, closed, connected and regular point set. A subset $K$ of a continuous curve $N$ is an open subset of $N$ provided $N-K$ is a closed, non-vacuous set.

$\ddagger$ I.e., there exists no connected subset in the plane which contains $A$ and $B$ and no point of $C$.

$\S$ If $P$ is a point of a point set $M$, then that maximal connected subset of $M$ determined by $P$ is the set of all points of $M$ that lie with $P$ in a connected subset of $M$.

\|Cf. Anna M. Mullikin, Certain theorems relating to plane connected point sets, these Transactions, vol. 24 (1922), pp. 144-162.

If A subset $N$ of a point set $M$ is said to separate two points $A$ and $B$ of $M-N$, if $M-N$ is the sum of two mutually exclusive sets $T$ and $U$ which contain $A$ and $B$ respectively, and neither of which contains a limit point of the other.
} 


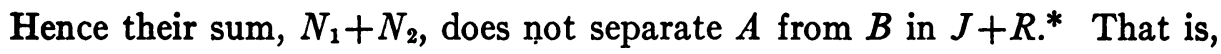
$A$ and $B$ are two points of the open subset $J+R-\left(N_{1}+N_{2}\right)$ of the continuous curve $J+R$, which are not separated in that open subset in the strong sense. Hence by Lemma $1, A$ and $B$ are not separated in that open subset in the weak sense. Accordingly, there exists a connected subset of $J+R-\left(N_{1}+N_{2}\right)$ which contains $A$ and $B$. As $N_{1}+N_{2}$ contains all points common to $J+R$ and $C$, a contradiction of the fact that $C$ separates the plane between $A$ and $B$ results.

\section{LEMMa 3. The point set $M$ described above is connected.}

Suppose $M$ is not connected. Then it is the sum of two sets $M_{1}$ and $M_{2}$ which are mutually separated. Clearly no $Q_{i}$ has points in both $M_{1}$ and $M_{2}$, as every $Q_{i}$ is connected. Hence each of the sets $M_{1}, M_{2}$ contains points of the set $P_{1}+P_{2}$.

Let

$$
\begin{aligned}
& M_{1} \times\left(P_{1}+P_{2}\right)=T_{1}, \\
& M_{2} \times\left(P_{1}+P_{2}\right)=T_{2} .
\end{aligned}
$$

$T_{1}$ and $T_{2}$ are mutually separated sets, since they are subsets of mutually separated sets, and

$$
T_{1}+T_{2}=P_{1}+P_{2} .
$$

If $m$ is a point of $P_{1}+P_{2}$ in $T_{1}$, say, then all those points of $P_{1}+P_{2}$ on the line $x=\xi_{1}$ which contains $m$ belong to $T_{1}$. For suppose that $q$ is a point of $P_{1}+P_{2}$ on $x=\xi_{1}$ belonging to $T_{2}$. Let the ordinate of $q$ be less than that of $m$. By Theorem 37 (p. 233) of S.E.C., there exists a continuum $C$ which contains no point of $P_{1}+P_{2}$ and which separates the plane between $m$ and $q$.

Denote the intersection of $x=\xi_{1}$ and the $x$-axis by $z$. Then $z$ is a limit point from the left, say, of end points of complementary intervals of $X$. Hence there exists an infinite sequence of distinct segments of the set $\left[e_{n}\right]$, viz., $\bar{e}_{1}, \bar{e}_{2}, \bar{e}_{3}, \cdots$, whose end points have $z$ as a sequential limit point, $\dagger$ and all of which lie to the left of $z$. Denote the corresponding elements of

* Cf. R. L. Moore, Concerning upper semi-continuous collections of continua which do not separate a given continuum, Proceedings of the National Academy of Sciences, vol. 10 (1924), pp. 356-360, Theorem 2.

† A point $P$ is a sequential limit point of a point set $K$ if every circle which encloses $P$ also encloses all except a finite number of points of $K$. 
the set $\left[s_{n}\right]$ by $\bar{s}_{1}, s_{2}, \bar{s}_{3}, \cdots$. Let $b$ be a point whose abscissa is less than that of $m$, and whose ordinate is the same as that of $m$. Let $d$ be a point which has the same abscissa as $b$ and the same ordinate as $q$. Denote the interior of the rectangle $b m q d b$ by $R$. By Lemma 2, there exists a subcontinuum $C^{\prime}$ of $C$ which contains points of the broken line $m b d q$ and of the straight line interval $m q$, and which, except for these points, is a subset of $R$.

Since $m$ and $q$ are not on $C^{\prime}$, and $C^{\prime}$ is closed, there exists a number $\eta<\xi_{1}$, such that if $r$ and $u$ are the intersections of $x=\eta$ with the straight line intervals $b m$ and $d q$, respectively, there are no points of $C^{\prime}$ on the straight line intervals $r m, u q$. Also, there exist two elements $\bar{s}_{i}$ and $\bar{s}_{j}$ of the set $\left[\bar{s}_{n}\right]$ such that the end points of $\bar{e}_{i}$ and $\bar{e}_{j}$ lie between the point on the $x$-axis whose abscissa is $\eta$ and the point $z$. Let $(f)$ and $(g)$ be abscissas of points in $\bar{e}_{i}$ and $\bar{e}_{j}$, respectively, and suppose $(f)<(g)$. There exists a subcontinuum $\bar{C}$ of $C^{\prime}$ which contains points of the lines $x=(f)$ and $x=(g)$, and which, except for these points, lies wholly between these two lines. As $\eta<(f)<(g)<\xi_{1}, \bar{C}$ lies wholly in $R$. Let the highest point of $\bar{C}$ on $x=(f)$ be $f$, and on $x=(g)$ be $g$, and let the projections of these two points on the $x$-axis be $F$ and $G$, respectively. Let the smallest ordinate possessed by any point on $\bar{C}$ be $\epsilon$, and let the intersections of $y=\epsilon$ with $x=(f)$ and $x=(g)$ be $\bar{f}$ and $\bar{g}$ respectively. Let those points of $P_{1}+P_{2}$ which lie within the rectangle $\bar{f} \bar{g} G F \bar{f}$ be denoted by $\bar{P}_{1}$. The set $\bar{P}_{1}$ is certainly not vacuous, for if $\rho$ is the abscissa of the right-hand end point of $\bar{e}_{i}$, there are points of $\bar{P}_{1}$ on $x=\rho$. Let $\bar{P}_{2}$ denote the set of points of $P_{1}+P_{2}$ which lie between $x=(f)$ and $x=(g)$, and above $\bar{C}$.

Let $w$ be any arc whose end points are $F$ and $G$ and which lies, except for $F$ and $G$, entirely below the $x$-axis. Then the arc $w$, together with the straight line intervals $f F$ and $g G$ and the continuum $\bar{C}$, forms a continuum $K$ which separates the plane between $\bar{P}_{1}$ and $\bar{P}_{2}$ and contains no point of $P_{1}+P_{2}$. Let $B$ denote the set of all those points of $P_{1}+P_{2}$ which lie in that complementary domain of $K$ which contains $\bar{P}_{1}$. The sets $H$. and $P_{1}+P_{2}-H$ are mutually separated. Let $H^{*}$ and $\left(P_{1}+P_{2}-H\right)^{*}$ denote the subsets of $S-a$ which correspond to $H$ and $P_{1}+P_{2}-H$ respectively under the correspondence $Z$ described above. It is easy to see that $a$ is not a limit point of $H$. Hence $a+\left(P_{1}+P_{2}-H\right)^{*}$ and $H^{*}$ are mutually separated. This is impossible, since $S$ is connected. Thus the supposition that $q$ is a point of $T_{2}$ leads to a contradiction and all points of $P_{1}+P_{2}$ on a line $x=\xi$ belong wholly to $T_{1}$, or to $T_{2}$, as the case may be.

Let the set of those points of $X$ which are the projections of points of 
$\tau_{i}(i=1,2)$ on the $x$-axis be denoted by $X_{i}$. Then $X_{1}$ and $X_{2}$ are mutually separated.

If one end point of a segment $e_{n}$ is in $X_{1}$, say, both end points are in $X_{1}$. Otherwise the point $P_{11 n}$ would be in $T_{1}$, say, and $P_{21 n}$ would be in $T_{2}$, which is impossible since $Q_{2^{n-1}}$ lies wholly in one of the sets $M_{1}, M_{2}$. For every $n$, add all points of $e_{n}$ to that set $X_{i}$ to which its end points belong. Denote the sets obtained by adding points to $X_{1}$ and $X_{2}$ in this way by $U_{1}$ and $U_{2}$, respectively. Then $U_{1}$ and $U_{2}$ are mutually separated, since no point of a segment $e_{n}$ is a limit point of points of the $x$-axis exterior to $e_{n}$. But this is impossible, since the set $U_{1}+U_{2}$ is identical with the set of all points in the interval $[0,1]$. Hence the supposition that $M$ is not connected leads to a contradiction.

Lemma 4. The point set $M$ is regular at every point of the set $P_{1}+P_{2}$.

Let $t$ be a point of $P_{1}+P_{2}$ which does not belong to any $Q_{i}$. Let $K_{1}$ be any circle with center at $t$. Let $W$ be a rectangle with center at $t$, lying wholly interior to $K_{1}$, with sides parallel to the coördinate axes and such that (1) the vertical sides of $W$ contain no points of $P_{1}+P_{2}$, (2) if $H$ is the height of $W$, the width of $W$ is such that no elements of the set $\left[s_{n}\right]$ have points within $W$ whose subscripts, $n$, do not satisfy the inequality $1 / 2^{n}<H / 4$, it being understood that this condition does not apply to the elements of $\left[s_{n}\right]$ in which the vertical sides lie, (3) the upper and lower bases of $W$ lie at a rational distance above the $x$-axis. Let $K_{2}$ be a circle concentric with $K_{1}$, lying interior to $W$, and not enclosing any points of those elements of the set $\left[Q_{n}\right]$ which have points on $W$. Such a circle exists, since $t$ is not a point or a limit point of any $Q_{i}$ and the diameters of the sets $\left[Q_{n}\right]$ approach zero as $n$ increases. Clearly no point of $P_{1}+P_{2}$ interior to $W$ is a limit point of the sum of all those sets of $\left[Q_{n}\right]$ which have points on $W$, unless it be a point of some such set.

Let $N$ be the set of all points of $P_{1}+P_{2}$ interior to $W$ or on the lower base of $W$, together with all sets $Q_{n}$ which lie wholly interior to $W$. Clearly $N$ is similar to the set $M$ and it may be shown to be connected by the same argument that was used to prove $M$ connected. As $K_{2}$ encloses only those points of $M$ which belong to $N, M$ is obviously regular at $t$.

If $t$ is a point of $P_{1}+P_{2}$ belonging to a set $Q_{i}$, select $W$ as before. Select $K_{2}$ so that it encloses no points of any set $Q_{n}$ excepting $Q_{i}$, which has points on $W$. Select $N$ as before. Since $Q_{j}$ is regular at $t$, there exists a circle $K_{3}$, concentric with $K_{1}$, such that all points of $Q_{i}$ interior to $K_{3}$ lie, with $t$, in a connected subset of $Q_{i}$ which lies wholly interior to $K_{1}$. Then the smaller 
of the two circles $K_{2}, K_{3}$ (or either of them, if they are identical) is clearly such that any point of $M$ within it lies, with $t$, in a connected subset of $M$ which lies wholly interior to $K_{1}$.

\section{II}

Consider a set $N$ constructed as follows: On the interval $[0,1]$ of the $x$-axis, let $X$ be a non-dense perfect set. Denote the point $\left(\frac{1}{2}, 1\right)$ by $P$. For any point $x$ of $X$, if $x$ is an end point of an interval complementary to $X$, assign the straight line interval $P x$ to a class $E_{1}$; otherwise to a class $E_{2}$. The set of all points on the $x y$-plane whose ordinates are rational and which lie on intervals of $E_{1}$ form a point set $N_{1}$, and the set of all points whose ordinates are irrational and which lie on intervals of $E_{2}$ form a set $N_{2}$. The set $N_{1}+N_{2}$ is connected, as it is identical, except for choice of coördinates, with the set $S$. Let the set $N_{1}+N_{2}$, together with the set of points symmetrical to it with respect to the $x$-axis, be denoted by $Q_{0}$. The set $Q_{0}$ is obviously connected. Let the point symmetrical to $P$ with respect to the $x$-axis be denoted by $P^{\prime}$.

As the intervals complementary to $X$ on the interval $[0,1]$ form a denumerable set, they can be ordered in a sequence $I_{1}, I_{2}, I_{3}, \ldots$. For every $n$, let $e_{1 n}$ and $e_{2 n}$ denote the end points of $I_{n}$, and let the set of points interior to the triangle $P e_{1 n} e_{2 n}$ be denoted by $s_{n}$. Let $Q_{k}^{1}, k=2^{n-1}, 2^{n-1}+1, \cdots$, $2^{n}-1$, be $2^{n-1}$ sets such that (1) for every $k, Q_{k}{ }^{1}$ is in one-to-one continuous correspondence with $Q_{0},(2)$ if $L_{i}$ is the line $y=i / 2^{n}$, where $i$ takes on all odd integral values between 0 and $2^{n}$, and $P_{1 i n}$ and $P_{2 i n}$ are the intersections of $y=i / 2^{n}$ with the straight line intervals $P e_{1 n}$ and $P e_{2 n}$, respectively, then for $k=2^{n-1}+\frac{1}{2}(i-1), Q_{k}^{1}$ (i) contains $P_{1 i n}$ and $P_{2 i n}$ and these points correspond, in the one-to-one continuous correspondence between this set and $Q_{0}$, to $P$ and $P^{\prime}$, respectively, and (ii) is symmetrical with respect to the line $y=i / 2^{n}$, has no limit points, except $P_{1 i n}$ and $P_{2 i n}$, that do not lie in $s_{n}$, and if $x$ is any point of it, the distance of $x$ from $L_{i}$ is less than $1 / 2^{n}$.

Denote the class each of whose elements is a set $Q_{i}^{1}(i=1,2, \cdots)$ by $\left[Q^{1}\right]^{1}$ and by $\left[Q^{1}\right]^{2}$ the class whose elements are symmetrical, with respect to the $x$-axis, to the elements of $\left[Q^{1}\right]^{1}$, and by $\left[Q^{1}\right]$ the class whose elements are the elements of $\left[Q^{1}\right]^{1}$ and $\left[Q^{1}\right]^{2}$.

Denote by $\left[Q^{2}\right]$ a class whose elements are constructed relative to all the elements of $\left[Q^{1}\right]$ just as the elements of $\left[Q^{1}\right]$ were constructed relative to $Q_{0}$. In general, denote by $\left[Q^{n}\right]$ a class whose elements are constructed relative to all the elements of $\left[Q^{n-1}\right]$ just as the elements of $\left[Q^{1}\right]$ were constructed relative to $Q_{0}$. 
Let $N$ be that point set which consists of all points of $Q_{0}$, as well as of all points which are contained in elements of the classes $\left[Q^{i}\right](i=1,2,3$, $\cdots$ ).

\section{THEOREM. The point set $N$ is a connected regular point set which contains no continuum.}

That $N$ is connected is evident. That $N$ is regular at $P$ and $P^{\prime}$ is easily shown from the fact that $Q_{0}$ is itself regular at these points. Similarly, every element of $\left[Q^{n}\right](n=1,2,3, \cdots)$ is regular at the points corresponding to $P$ and $P^{\prime}$. The set $N-\left(P+P^{\prime}\right)$ is therefore a set of the same type as the set $M$ described above, and is therefore regular at all points of the set $Q_{0}-\left(P+P^{\prime}\right)$ by Lemma 4 .

For every $n$, arrange the elements of the class $\left[Q^{n}\right]$ in a sequence $Q_{1}{ }^{n}$, $Q_{2}{ }^{n}, Q_{3}{ }^{n}, \cdots$. If $Q_{2}{ }^{1}$ is any element of $\left[Q^{1}\right]$, it has been shown already that $N$ is regular at the points of $Q_{i}^{1}$ corresponding to $P$ and $P^{\prime}$. As for the other points of $Q_{i}^{1}$, none of these is a limit point of the other elements of $\left[Q^{1}\right]$, and as $Q_{i}{ }^{1}$ is a set of the same type as the set $Q_{0}, N$ is regular at all these points. As for any element $Q_{i}{ }^{n}$ of any class $\left[Q^{n}\right]$, a similar argument holds, since no one of these sets contains, except for the points corresponding to $P$ and $P^{\prime}$, a limit point of any element or set of elements of the classes $\left[Q^{i}\right](i=1,2,3, \cdots, n-1)$ or of $\left[Q^{n}\right]$ distinct from itself. Hence $N$ is a regular point set.

The point set $N$ contains no continuum. For suppose it does contain a continuum $K$. Then $K$ contains a point, $A$, of an element $Q_{i}{ }^{i}$ of the class [ $\left.Q^{i}\right]$, say, where $A$ is not one of the two points of $Q_{i}{ }^{i}$ corresponding to $P$ and $P^{\prime}$ of $Q_{0}$. For the set of points in sets $Q_{i}{ }^{i}(i=1,2, \cdots, j=1,2, \cdots)$ corresponding to $P$ and $P^{\prime}$ of $Q_{0}$ is clearly denumerable and no continuum, nor indeed any connected set, can consist of a denumerable set of points. If $d$ and $f$ are the points of $Q_{i}^{j}$ corresponding to $P$ and $P^{\prime}$ in $Q_{0}$, it is easily shown by Theorem 1 of the paper of Miss Mullikin referred to above that $K$ has a subcontinuum, $K_{0}$, which contains $A$, but does not contain $d$ or $f$, nor any point of any other element of the class $\left[Q^{i}\right]$, nor any point of an element of a class $\left[Q^{n}\right]$ for which $n<j$. The continuum $K_{0}$ cannot lie wholly in $Q_{i}^{j}$ since the latter set contains no continuum. Hence $K_{0}$ contains a point $B$ of some set $Q_{k}^{m}$ such that $B$ is not a point of this set corresponding to $P$ or $P^{\prime}$ in $Q_{0}$, and such that $m>j$. Then $K_{0}$ contains a subcontinuum $K_{1}$ which contains $B$ but no point of an element of a class $\left[Q^{n}\right]$ for which $n<m$. By repetition of this argument, the existence of a sequence of continua 
$K_{1}, K_{2}, K_{8}, \cdots$ may be shown such that for every positive integer $q, K_{q+1}$ is a subset of $K_{q}$, and such that $K_{q}$ contains no point of any element of a class [Q] for which $n<q$. The sets $K_{1}, K_{2}, K_{3}, \cdots$ have in common at least one point $P$, and this point does not belong to any element of a set $\left[Q^{n}\right]$. Thus the supposition that $N$ contains a continuum $K$ is shown to lead to a contradiction, and the theorem is proved.

Oho State Untverstiy,

Columbus, OHIо 